\title{
A Study of the Antenna Effect of Photovoltaic Modules
}

\author{
M. Drapalik ${ }^{1}$, J. Schmid ${ }^{1}$, E. Kancsar ${ }^{1}$, V. Schlosser ${ }^{1}$, G. Klinger ${ }^{2}$ \\ ${ }^{1}$ University of Vienna, Faculty of Physics \\ Department of Electronic Properties of Materials \\ Strudlhofgasse 4, A-1090 Vienna (Austria) \\ phone number: +43 14277 51428, e-mail: markus.drapalik@univie.ac.at, viktor.schlosser@univie.ac.at \\ ${ }^{2}$ University of Vienna, Faculty of Geosciences, Geography and Astronomy \\ Department of Meteorology and Geophysics \\ Althanstraße 14, A-1090 Vienna (Austria) \\ phone number: +43 14277537 23, e-mail: gerhard.klinger@univie.ac.at
}

\begin{abstract}
We have investigated the reaction of photovoltaic cells to external electromagnetic fields in a frequency range of $10 \mathrm{~Hz}$ to $1 \mathrm{GHz}$. Outdoor recordings of received RF noise from the ambient were sampled for different solar cells and modules and compared with the reception of a simple whip antenna which represents a monopole for electromagnetic fields. Additionally in the laboratory well defined signals were generated and their reception was investigated depending on geometrical factors of the cells, such as orientation, size and distance. Two antenna models, namely dipole and patch configuration, are compared with the experimental findings. Simulations for the effect of modulated signals on solar cells were made as well. It was found that the gain in the investigated frequency range increases essentially linear with the cell area similar to patch antennas. A crystalline silicon cell of $100 \mathrm{~cm} 2$ has almost the same signal amplification as an $83 \mathrm{~cm}$ long whip antenna remaining coarsely constant over the whole frequency range. For narrow frequency bands however the signal attenuation or amplification is strongly modified by the dipole behavior caused by the front metal grid. Simulation shows that demodulation effects are biggest around the MPP, possibly affecting subsequent power conditioning units.
\end{abstract}

\section{Key words}

Electromagnetic Interference, RF signals, photovoltaic cell, solar power generator

\section{Introduction}

Rising interest and increasing subsidies in the last years led to an exponential increase in the installed capacity of photovoltaic power generators. Numerous solar parks are already in operation under construction or are planned in the European Union, which cover areas of several thousand square meters. The adaptation of solar modules for building integration results in large facades covered with interconnected electronic devices. It can be said that photovoltaic power generators with an active area of over $1000 \mathrm{~m}^{2}$ are no rarity anymore.
Considering the huge area covered with electronic devices with a complex AC behavior, which is not fully investigated yet, a reaction to external electromagnetic fields must be expected. Given the interaction of the solar modules with power electronic and the power grid, yet unknown problems of electromagnetic compliance may arise. [1-3]

Several possible mechanisms of the interaction between photovoltaic modules and electromagnetic fields or radiation have to be taken into account: radiation may be received or reflected by the semiconducting devices, or may be conducted to the subsequent power conditioning electronics. In opposite direction system distortion can be emitted to air by solar modules. Different ambient sources, such as vibrations, light fluctuations or internal noise [4], can additionally generate $\mathrm{AC}$ currents in the photovoltaic power plant thus increasing the total electric noise level in the system.

The goal of our present work is a qualitative understanding of the reaction of photovoltaic cells to external electromagnetic fields. Different cells were investigated in a frequency range of $10 \mathrm{~Hz}$ to $1 \mathrm{GHz}$ which covers a great portion of today's electromagnetic emission sources. An example is shown in fig.1. The influence of various solar cell design parameters like front metal grid and cell dimensions were examined.

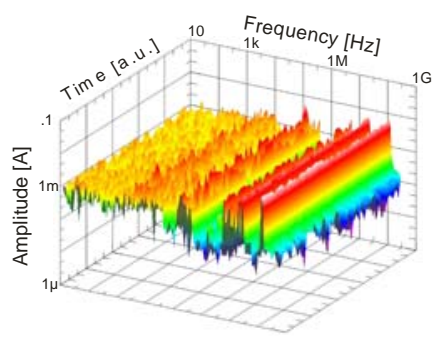

Fig 1: Current noise spectra due to ambient electromagnetic radiation of a $10 \mathrm{~W}$ solar module 


\section{Theory}

The general layout of most solar cells and modules suggests using two antenna models for the theoretical description of solar cells as a receiver or emitter of $\mathrm{RF}$ radiation.

The first approach assumes that the front grid of the currently most widespread c-Si cells can be modeled by an array of dipoles. The dipoles given by the highly conductive grid are electrically isolated from the back contact. The insulator is determined by the dielectric properties of the semiconductor and the cell's thickness. In this model the back contact simply acts as a reflector for the electromagnetic wave. Since the ideally negligible dielectric losses between front and back contact are essential for the application of this model only thick semiconducting layers present solely in crystalline silicon cells will sufficiently well match the condition of good electrical isolation. [5] Depending on the geometry and number of dipoles one or multiple band-pass frequencies can occur. For a single dipole the impedance can be found using well known equations [5]. For interconnected, multiple dipoles an array factor modifying the radiation pattern of the single dipole can be found for simple geometrical configurations. For example for the $\mathrm{H}$-pattern front grid with $2 \mathrm{~N}$ fingers at a distance $\mathrm{D}$, the array factor $\mathrm{AF}$ in spherical coordinates is:

$A F_{\text {total }}=\frac{\sin (2 k d \cos \theta)}{2 \sin (k d \cos \theta)} \frac{\sin \left(\frac{N}{2} k D(\sin \theta \cos \phi+1)\right)}{N \sin \left(\frac{1}{2} k D(\sin \theta \cos \phi+1)\right)}$

where $\mathrm{k}$ is the wavenumber.

In the second approach the front contact is assumed to form an electrically homogenous area - the patch separated from the reference or ground potential assigned to the back contact. In this case the dielectric in between need not be assumed lossless and can be described by an effective permittivity (dielectric constant) which generally is complex. For model calculations most often the imaginary part however is neglected. Thus the reception properties depend on the dimensions of the cell and the relative permittivity of the substrate. This model may give a good representation of thin film cells. [6] The transfer function typically has only one very broadband band-pass characteristics. The resonance frequency, $v_{\text {res }}$, can be roughly estimated using the simple transfer line model, which gives:

$L_{e f f} \cdot \nu_{r e s}=\frac{c}{2 \sqrt{\epsilon_{e f f}}}$

where $c$ is the velocity of light. The effective length $\mathrm{L}_{\text {eff }}$ and the effective relative permittivity $\varepsilon_{\text {eff }}$ can be calculated using empirical formulae for example given in [6].

Summarizing, the impedance of a dipole array is dependent on the number of dipoles, their length and their spacing, while the impedance of a patch antenna depends on its area and dielectric.

\section{Experimental}

In the laboratory a well defined signal (by means of amplitude, frequency and phase) was emitted by a dipole antenna and the received signal was analyzed at the samples terminals. The setup for the performed experiments consists of a lock-in amplifier with a built-in sine wave generator, a signal generator, a dipole antenna and the devices under test (DUT). The sending antenna was mounted onto the pen holder of a plotter in order to vary the position between sender and receiver.

In order to take advantage of the high sensitivity of the lock-in amplifier detection with an upper frequency limit of $100 \mathrm{kHz}$, two different operation modes were used:

Below $100 \mathrm{kHz}$, the emitted sinusoidal signal is directly generated by the signal generator with which the lock-in amplifier synchronizes.

For higher frequencies the internal generator of the lock in amplifier is used to produce a modulation signal around $1 \mathrm{kHz}$. The external function generator produces a carrier signal which is amplitude modulated by the lock in amplifiers output. This mode is used for frequencies up to $20 \mathrm{MHz}$.

Antenna and DUT are placed in an electromagnetically shielded compartment, which has the size of $60 \times 51 \times 45 \mathrm{~cm}^{3}$. The shielding is achieved by the use of grounded $1 \mathrm{~mm}$ steel plates.

In the "natural" electromagnetic ambience given by the numerous RF emitting sources, indoors as well as outdoors, the input of a $83 \mathrm{~cm}$ long whip antenna (resonance frequency approximately $200 \mathrm{MHz}$ ) was simultaneously recorded with the input of different solar cells and panels using a $2 \mathrm{GSs}^{-1}$ sampling oscilloscope. Both devices, antenna and solar cell, were connected via a $1 \mathrm{~m}$ long coaxial transmission cable to the high impedance input of the oscilloscope without a matching circuit. The time domain signals were Fourier transformed and the ratio of the squared amplitudes up to $1 \mathrm{GHz}$ was averaged over the time. Since only "natural" sources were received several gaps in the frequency range occurred were the signal levels remains permanently close or below the measurement resolution of $80 \mu \mathrm{V}$. In these cases the derived ratio leads to large deviations.

\section{Results and Discussion}

\section{A. Sender-Receiver Geometry}

Several cells were examined in different orientations towards the sending antenna. If the antenna length is parallel to the $\mathrm{x}$-axis, the directions were the following:

- cell in plane with the sending antenna (in the $x-y-$ plane)

- cell in the $x-y-p l a n e$, rotated by $90^{\circ}$

- cell in the x-z-plane

- cell in the y-z-plane 
The arrangement is shown on top of fig.2 together with the results for a $100 \mathrm{~cm}^{2} \mathrm{c}-\mathrm{Si}$ cell with ?? fingers $/ \mathrm{cm}$ is below. The cell in plane with the sending antenna, with its fingers aligned with the dipole axis (in the following referred to as parallel alignment), responded strongest to the external field at low frequencies. The cell in the x-zplane, which is also in parallel alignment, showed the second best gain, while the other two configurations showed a $12 \mathrm{~dB}$ weaker reaction. Contrary to the findings of Wada et al. [1], who assumed the bus bars to give the major contribution and ignored the finger influence, in this case the alignment of the fingers parallel to the dipole leads to higher amplitudes than the parallel alignment of the bus bars.
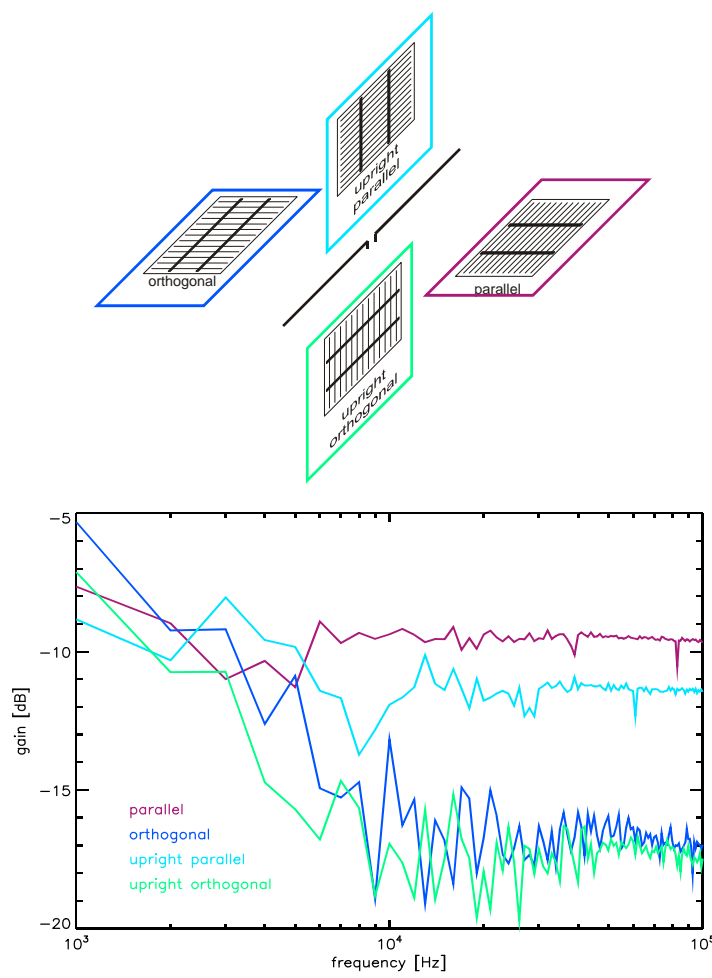

Fig 2: Gain per orientation towards sending antenna. The configuration is shown on top.

\section{B. Size Dependency}

Comparison of the gain compared to a monopole antenna of differently sized crystalline silicon solar cells shows that the gain increases linearly with the area of a cell. Fig. 3 shows the gain per area for four DUTs, which is very similar for three of them at low frequencies. Only the gain of the DUT drawn in red is approximately $3 \mathrm{~dB}$ weaker. In contrast to the rectangular shape of the other cells this cell has a circular shape which could potentially explain the weaker amplification. At frequencies above $10 \mathrm{kHz}$ differences between the cells virtually increase most likely due to the lack of strong signals, the tendency however remains similar.

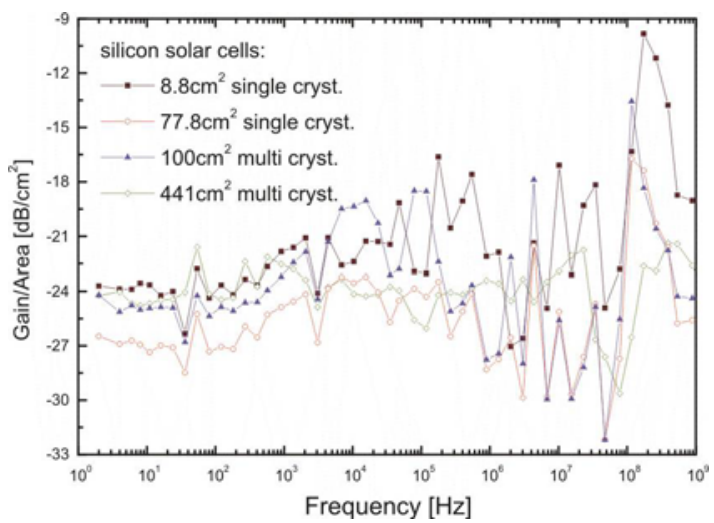

Fig. 3: Gain per area for four solar cells

The difference between two samples whose measured signal intensity differed by a factor of two could be explained comparing their dipole line density. The DUT with the higher intensity had a twice as narrow front grid as the DUT with the weaker signal. Roughly a c-Si cell with an area somewhat larger than $100 \mathrm{~cm}^{2}$ will reach at least zero gain compared to the simple whip antenna throughout the whole frequency range.

\section{Comparison with Patch}

The signals received by an $8.8 \mathrm{~cm}^{2}$ and a $100 \mathrm{~cm}^{2}$ sized crystalline solar cell were compared with equally sized patches of copper covered hard paper.

As can be seen in fig. 4, the measured gains differ very little below $2 \mathrm{MHz}$. Above this value the smaller cell shows more similarities with the patch than the bigger cell. The theoretical resonance frequency for the $100 \mathrm{~cm}^{2}$ patch is $\sim 450 \mathrm{MHz}$, for the $8.8 \mathrm{~cm}^{2}$ patch $1.35 \mathrm{GHz}$, while measurements went up to $20 \mathrm{MHz}$.

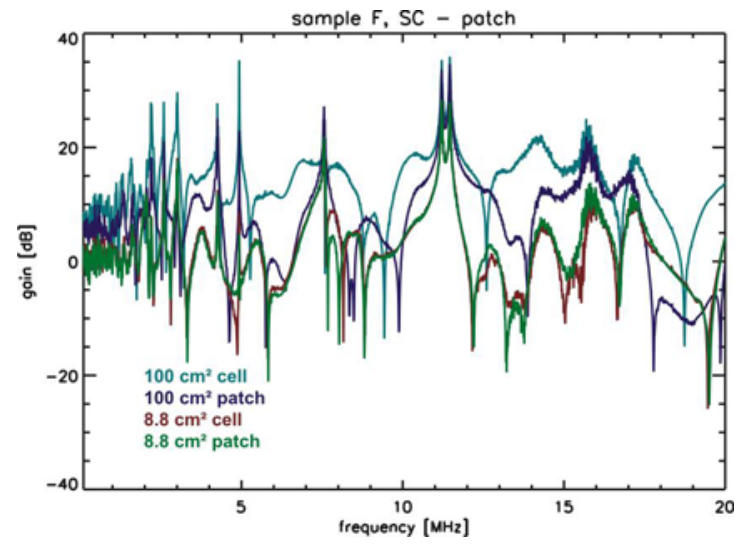

Fig. 4: Comparison of c-Si solar cells with equally sized patches

\section{Comparison between c-Si and a-Si}

Comparing the signals of an amorphous silicon mini module with those of a crystalline silicon module showed that a-Si cells amplify electromagnetic radiation better than c-Si devices. As can be seen in fig. 5 , the gain of the amorphous module is at least $6 \mathrm{~dB}$ higher than that of the 
crystalline module, although its area is less than $1 / 3$ of the c-Si module.

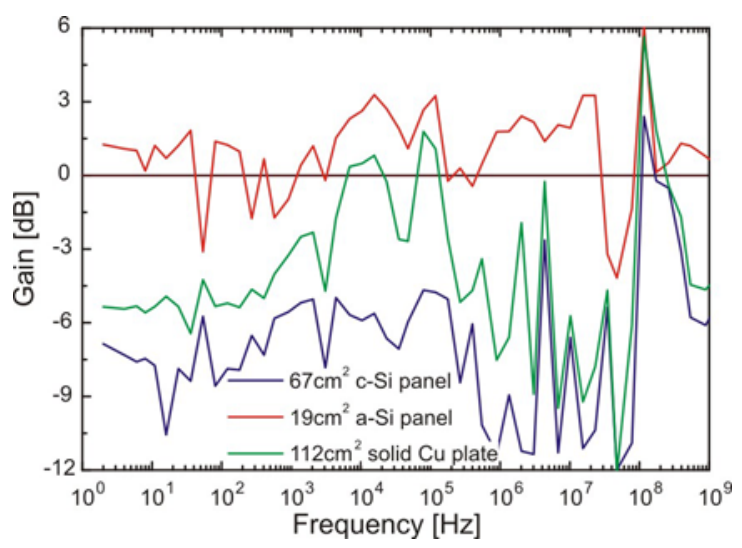

Fig. 5: Gain of a crystalline and an amorphous silicon solar panel, as well as a solid copper plate for comparison.

\section{Simulation}

Although high frequency noise introduced by photovoltaic modules is fast attenuated during the DC optimized power transmission demodulated radio frequency signals with sufficient lower frequencies can be transmitted over long distances. The demodulation of radio signals is caused by the intrinsic non-linear currentvoltage characteristic of any photovoltaic device. In order to get a rough estimate of the size of signal demodulation in a photovoltaic solar cell a simple simulation was performed with the simulation program Qucs [7]. The input parameters of the photovoltaic device were taken from the experimental findings of a small mc-Si cell. For $\mathrm{T}=300 \mathrm{~K}$ a single diode assumption with the diode ideality factor $n=2$ was sufficient for the investigated range. Therefore an equivalent circuit of the solar cell composed of discrete elements was assumed. The elements to compose the circuit are as follows: a diode with a saturation current $I_{0}=1 \mu \mathrm{A}$, a shunt resistor $R_{\mathrm{SH}}=5500 \Omega$, a series resistor, $R_{\mathrm{S}}=0.3 \Omega$. and an ideal current source for the light generated current $I_{\mathrm{ph}}$. Under artificial illumination with approx. $300 \mathrm{Wm}^{-2}, I_{\mathrm{ph}}=18.6 \mathrm{~mA}$ was observed. For the model calculations the cell was assumed to be terminated with a mere ohmic load which was varied between $1 \Omega$ and $1 \mathrm{k} \Omega$. In the simulation an amplitude modulated current source was added in parallel to the DC circuit. The rectangular on-off signal was modulated on the carrier wave by 100 per cent. The carrier amplitude was chosen to be 10 per cent of $I_{\mathrm{ph}}$. For three values of the load resistor the demodulated voltage was calculated. The values for these load resistors, $R_{\mathrm{L}}$ were $2.35 \Omega, 23.5 \Omega$ and $235 \Omega$ respectively which correspond to near short circuit conditions in the first case, operating the cell at its maximum power point at $23.5 \Omega$ and a condition close to open circuit for $235 \Omega$. The effect of the selected simulation condition on the static DC current voltage characteristic $\mathrm{I}_{\mathrm{DC}}(\mathrm{V})$ of the cell are illustrated by fig. 6 .

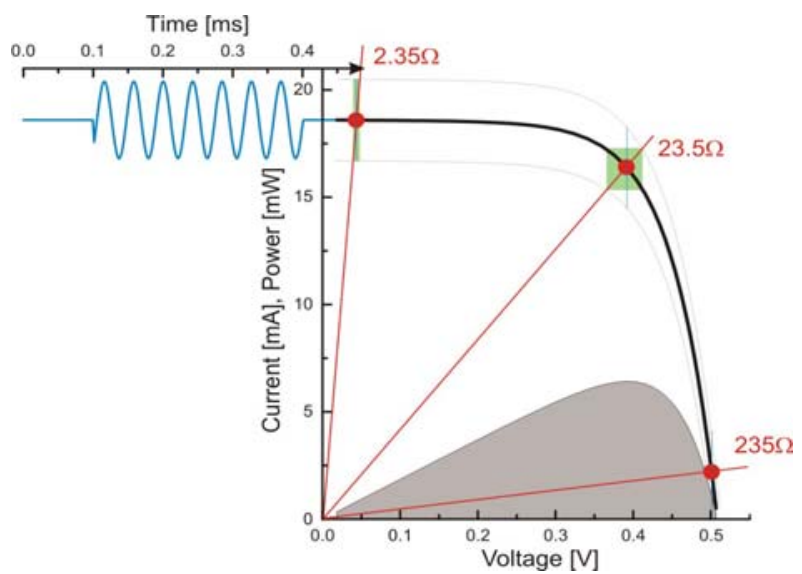

Fig. 6: The demodulation effect of a mc-Si solar cell. For details refer to text.

The $I_{\mathrm{DC}}(V)$ curve (black line) oscillates within the limits given by the grey dashed lines while the modulated signal is high which was visualized as blue line (current vrs. time) in the upper left graph of fig. 6.. The three load resistors are represented by red lines and their intersection with the $I_{\mathrm{DC}}(V)$ curve is marked by red circles. The light green rectangle at the working points is determined by the intersection of the load resistor with the oscillation limits (dashed grey lines) of the current voltage curve of the device. The non-linear characteristics causes the working point to be offset from the centre of the rectangle in the horizontal voltage direction thus causing an asymmetric voltage oscillation which basically gives the demodulated signal. The results of our simulation are summarized in table 1. Here the demodulated voltage $V_{\mathrm{dem}}$ is the horizontal shift of the rectangle's centre with respect to the $\mathrm{DC}$ value of the voltage $V_{\mathrm{DC}}$ at the working point.

\begin{tabular}{lllll}
\hline$R_{\mathrm{L}}[\Omega]$ & $I_{\mathrm{DC}}[\mathrm{mA}]$ & $V_{\mathrm{DC}}[\mathrm{mV}]$ & $V_{\mathrm{dem}}[\mathrm{mV}]$ & $\left|V_{\mathrm{dem}} / V_{\mathrm{DC}}\right|[\%]$ \\
\hline 2.35 & 18.59 & 43.69 & +0.04 & 0.09 \\
23.5 & 16.52 & 388.25 & -12.26 & 3.16 \\
235 & 2.13 & 501.08 & -0.10 & 0.02 \\
\hline
\end{tabular}

Table 1: Summarised results of the model calculations

The demodulation effect is small in both absolute and relative size at the both ends of the $I_{\mathrm{DC}}(V)$ characteristics. Close to short circuit current conditions the linear $R_{\mathrm{SH}}$ element of the cell is dominant thus reducing the demodulation efficiency. Near open circuit the current is significantly reduced thus resulting in a small demodulated signal. Demodulation however is large around the maximum power point of the cell were the current is still high and the non-linearity of the device is pronounced. Unfortunately this undesired origin of signal distortion contributes most during efficient power generation of a solar power generator which severely can affect the subsequent power conditioning units.

In order to optimise energy yield maximum power tracker are preferentially connected to the output of photovoltaic arrays. The feed back loop of maximum power tracking requires the pick up of the incoming signals by means of voltage and current delivered from 
the solar array in order to adjust the duty cycle of the switch in the converter. The stability of any control loop essentially depends on the integrity of the picked up signals. Noisy signals can lead to temporal or permanent malfunctions of the control loop. The simple solution to integrate the measured input values has to be carefully considered against the desired response time of the control loop. In this context low frequency noise in general and demodulated signals in particular have to be considered.

\section{Conclusions}

Our measurements showed that, for crystalline silicon cells, the reception of electromagnetic radiation depends linearly on the cell area, at least at low frequencies (below $10 \mathrm{MHz}$ ). The front grid can have significant influence depending on the line density. Comparison with a metal patch showed very good agreement for a small cell, less for a $100 \mathrm{~cm}^{2}$ cell.

This leads to the conclusion that the patch antenna model may only be used to roughly describe the reception behavior of crystalline silicon solar cells. This is likely due to the fact, that the front side is covered by a conductive grid rather than by a homogenous patch. Corrections following a dipole model have to be applied to cover the front grid influence.

Generally thin a-Si film cells amplify RF signals and hence EM noise better than crystalline silicon cells. Their structure strongly resembles that of microstrip patch antennas, because of its similarities: a dielectric substrate (eg. a-Si) is bounded by two conductive plates (the front and back contacts). Accordingly, their behavior may be described using a patch antenna model only which covers the lossy dielectric.

For practical applications it should be noted that the $19 \mathrm{~cm}^{2}$ a-Si panel received more noise than the $83 \mathrm{~cm}$ reference dipole. Given a photovoltaic power generator with an output of $1 \mathrm{kWp}$ a 500 times higher gain may be expected leading to significant output power distortion at the terminals of the photovoltaic assembly. From here it will be lead to the input of the power conditioning unit. Although it could be expected that attenuation takes place with increasing cable length no appropriate guidelines or regulations presently exist for that part of a photovoltaic power generator.

Following the reciprocity theorem, a solar cell array may also emit significant amounts of undesired electromagnetic radiation if it is excited by unfiltered noise in the power electronics, light fluctuations or vibrations.

The radiation or absorption in certain frequency bands may cause severe EMC issues. Depending on the size of single cells technical appliances i.e. for mobile communication, GPS or WLAN may be jammed.
In figure 3 an increased gain can be seen around the range from $30-300 \mathrm{MHz}$. This range should be paid additional attention since biological systems show increased reaction to electromagnetic radiation in this region.

Simulation shows that signal demodulation has the greatest effect close to the working point of solar cells, which may affect subsequent power conditioning units. This should be considered in the design of MPP-tracking algorithms.

\section{References}

[1] Wada, T., Mori, T., Tokuda, M., Suenaga, S., Igarashi, H., Proc. of the International Symposium on EMC, 2005, 1, 112.

[2] Di Piazza, M. C., Serporta, C., Tinè, G., Vitale, G., Proc. of IEEE International Conference on Industrial Technology, 2004, 2, 672.

[3] Igarachi, H., Suenaga, S., Proc. of the $31^{\text {st }}$ IEEE Photovoltaic Specialist Conference, 2005, 1820.

[4] Mallette, L. A., Phillips, R. L., Appl. Optics, 1978, 17, 1780 .

[5] Christodoulou, G, Wahid, P., Fundamentals of Antennas: Concepts and Applications. SPIE - The International Society for Optical Engineering, 2001

[6] Chang, K., Handbook of RF/microwave components and engineering. John Wiley \& Sons, Hoboken, New Jersey, 2003

[7] Quite universal circuit simulator at http://qucs.sourceforge.net/index.html 